Ger J Exerc Sport Res 2022 · 52:186-192 https://doi.org/10.1007/s12662-021-00754-2 Eingegangen: 10. Mai 2021

Angenommen: 24. August 2021

Online publiziert: 23. September 2021

(c) Der/die Autor(en) 2021

\section{Clemens Töpfer ${ }^{1}$ (D) Johannes Jaunig ${ }^{2} \cdot J^{\prime}$ Johannes Carl}

${ }^{1}$ Institut für Sportwissenschaft, Friedrich-Schiller-Universität Jena, Jena, Deutschland ${ }^{2}$ Institut für Bewegungswissenschaften, Sport und Gesundheit, Karl-Franzens-Universität Graz, Graz, Österreich

${ }^{3}$ Department für Sportwissenschaft und Sport, Friedrich-Alexander-Universität Erlangen-Nürnberg, Erlangen, Deutschland

\title{
Physical Literacy - to be discussed: eine Perspektive aus Sicht der deutschsprachigen Sportwissenschaft
}

Physical Literacy hat sich zu einem global verbreiteten Konzept entwickelt. Gerade in der internationalen Forschung schafft Physical Literacy einen integrierenden Ansatz für inhaltliche Anknüpfungspunkte und Vergleichbarkeit zwischen Studien aus verschiedenen Ländern. Physical Literacy bietet in seiner ursprünglichen Konzeption eine ganzheitliche („holistic“) Sichtweise auf personale Voraussetzungen und Kompetenzen in Bewegung und Sport (u.a. Edwards, Bryant, Keegan, Morgan, \& Jones, 2017; Holler et al., 2021; Whitehead, 2001). An das Verständnis von Whitehead anknüpfend, definiert die International Physical Literacy Association (IPLA) Physical Literacy wie folgt: „Physical literacy can be described as the motivation, confidence, physical competence, knowledge and understanding to value and take responsibility for engagement in physical activities for life" (IPLA, 2017).

Mit dem vorliegenden Beitrag möchten wir in der deutschsprachigen Sportwissenschaft zur Diskussion um das Konzept der Physical Literacy anregen. Im ersten Teil soll dabei aufgezeigt werden, wie das Konzept der Physical Literacy aktuell sowohl international als auch im

\footnotetext{
1 Wir möchten hier und an anderen Stellen unseres Beitrags bewusst die englische Schreibweise stehen lassen und keine deutschsprachigen Synonyme nutzen, da sie mitunter zu Fehlinterpretationen führen.
}

deutschsprachigen Raum rezipiert wird. Im zweiten Teil setzen wir an diesen Beobachtungen an und stellen Herausforderungen und Chancen zur Diskussion, warum sich die deutschsprachige Sportwissenschaft intensiver mit Physical Literacy beschäftigen sollte.

\section{Das Phänomen Physical Literacy: eine Moment- aufnahme}

\section{Physical Literacy international}

Mit der Veröffentlichung von Margaret Whiteheads Gedanken zu einer Physical Literacy (Whitehead, 2001) hat sich in den letzten 20 Jahren die Idee der Physical Literacy zu einem weitverbreiteten Konzept entwickelt. Dabei reicht die namentliche Nennung einer Physical Literacy jedoch bis in das frühe 20. Jahrhundert zurück (Bailey, 2020). Allerdings haben die Veröffentlichungen von Whitehead gewissermaßen den Weg dafür geebnet, dass Physical Literacy globale Verbreitung gefunden hat (Cairney, Kiez, Roetert, \& Kriellaars, 2019a) und speziell ab den 2010er Jahren eine rasante $\mathrm{Zu}$ nahme an Publikationen diesbezüglich erkennbar ist (Bailey, 2020). Jüngere Literaturreviews dokumentieren zusätzlich die deutlich zunehmende Aufmerksamkeit und Akzeptanz von Physical Literacy in der internationalen wissenschaftlichen Landschaft (u. a. Cornish et al., 2020; Ed- wards et al., 2017; Edwards et al., 2018; Pushkarenko, Causgrove Dunn, \& Wohlers, 2020). Zudem wird die Relevanz von Physical Literacy in fünf internationalen Themenheften („Special Issues“) hervorgehoben (u. a. Cairney, 2020; Chen \& Sun, 2015; Dudley, Cairney, \& Goodway, 2019; Durden-Myers \& Whitehead, 2018; Naylor \& Temple, 2018).

Obgleich Physical Literacy in den vergangenen Jahren viel Beachtung gefunden hat, wurde das Konzept immer wieder verschieden interpretiert (Edwards et al., 2017; Jurbala, 2015). Damit verbunden stehen auch immer Bemühungen, das Konzept der Physical Literacy vor Fehldeutungen zu bewahren und weiterzuentwickeln (u. a. Dudley, Cairney, Wainwright, Kriellaars, \& Mitchell, 2017). Um Physical Literacy insgesamt klarer fassen zu können, wurde in der internationalen Forschung mehrfach versucht, Physical Literacy konzeptionell $\mathrm{zu}$ systematisieren (u. a. Edwards et al., 2017) und empirisch zu operationalisieren (Cairney, Dudley, Kwan, Bulten, \& Kriellaars, 2019b; Gunnell, Longmuir, Barnes, Belanger, \& Tremblay, 2018). Auf Basis des Testinstruments "Canadian Assessment of Physical Literacy" (CAPL) konnten Gunnell et al. (2018, S. 133) beispielsweise faktorenanalytisch vier Bereiche voneinander abgrenzen: Daily Behaviour, Motivation \& Confidence, Physical Competence, Knowledge \& Understanding. 
Auch gesellschaftlich und politisch hat sich Physical Literacy zu einer weitreichenden Leitidee entwickelt. So hatte zuletzt auch die UNESCO (2015) Physical Literacy als ein relevantes Konzept zur Bewegungs- und Gesundheitsförderung in der Gesellschaft hervorgehoben. Zusätzlich haben sich in verschiedenen (insbesondere anglophonen) Ländern Organisationen auf die besondere Förderung von Physical Literacy fokussiert. In Kanada ist beispielsweise 2015 das „Physical Literacy Consensus Statement" aus verschiedenen Initiativen hervorgegangen (Tremblay et al., 2018). Auch Australien hat zuletzt ein "Australian Physical Literacy Framework" veröffentlicht, in dem Physical Literacy zur nationalen Agenda erklärt wurde (Scott, Hill, Barwood, \& Penney, 2020). In den USA wird Physical Literacy mittlerweile sogar als zentrale Leitidee in Lehrplänen des Sportunterrichts erachtet (Roetert \& MacDonald, 2015).

Fassen wir die aktuellen Befunde zur Physical Literacy zusammen, so wird deutlich, dass Physical Literacy - etwas umgangssprachlich ausgedrückt - „im Trend“ liegt. Es ist davon auszugehen, dass diese Dynamik im internationalen Raum einen Indikator für die zukünftige Entwicklung des Physical-LiteracyKonzepts darstellt.

\section{Physical Literacy im deutsch- sprachigen Raum}

Während Physical Literacy international schon relativ weit verbreitet ist, wurde das Konzept im deutschsprachigen Raum in den letzten Jahren nur vereinzelt aufgegriffen und dabei aus verschiedenen Perspektiven rezipiert.

Aus sportpädagogischer Sicht wurde Physical Literacy zuletzt für den Sportunterricht ausgedeutet. So nutzen beispielsweise Hummel und Krüger (2020) das Konzept der Physical Literacy als Begründungsrahmen für eine „neue körperliche Grundbildung“. Gleich zu Beginn grenzen sie sich dabei von einer sogenannten „Sportliteralität“ ab und verweisen zum Beispiel auf Veröffentlichungen von Schierz und Miethling (2017), in denen es um „Bildung über Sport“ geht (Hummel \& Krüger, 2020,
S. 295). In ihrem Verständnis jedoch „zielt Physical Literacy auf Bildung und Erziehung durch und mit Hilfe von Bewegung, Spiel und Sport“ab (ebd.). Hummel und Krüger (2020) verfolgen mit ihrem Entwurf einerseits die „bestmögliche Förderung motorischer Fähigkeiten und die Entwicklung des individuellen Bewegungskönnens" (S. 299) und andererseits die „Reflexivität und das Verstehen des eigenen Bewegungshandelns sowie aufgeklärte Bewusstseinsbildung über Sinn und Zweck, Wert und Bedeutung körperlicher Bildung“ (ebd.). Neben den Impulsen von Hummel und Krüger (2020) wird in einem aktuellen Projekt auch daran gearbeitet, Physical Literacy im Rahmen von Curricula der Lehramtsausbildung zu implementieren und den Sportunterricht zu innovieren (Knisel \& Bremer, 2021). Darin wird hervorgehoben, dass Physical Literacy einen modernen Sportunterricht verkörpert, „der nicht nur wie bislang die Vermittlung von Sportarten in den entsprechenden Bewegungsfeldern fokussiert, sondern das Wissen und das Verstehen über den Körper und dessen Funktionen sowie über Bewegung im sozialen Kontext in den Mittelpunkt stellt" (Knisel \& Bremer, 2021).

Im deutschsprachigen Raum wurde Physical Literacy zuletzt auch verstärkt mit Gesundheitskompetenz in Verbindung gebracht. Hier sind zum einen das Modell der sportbezogenen Gesundheitskompetenz (SGK; Töpfer \& Sygusch, 2014; Töpfer, 2019) und zum anderen das Modell der bewegungsbezogenen Gesundheitskompetenz (BGK; Pfeifer, Sudeck, Geidl, \& Tallner, 2013; Sudeck \& Pfeifer, 2016) zu nennen.

Das SGK-Modell wurde vorrangig vor dem Hintergrund der sportpädagogischen Gesundheits- und Kompetenzdiskussion konzipiert. Es beruht maßgeblich auf der Leitidee der Handlungsfähigkeit, welche das selbstbestimmte und verantwortungsvolle Handeln von Kindern und Jugendlichen im Sport in den Mittelpunkt stellt (u.a. Gogoll \& Kurz, 2013). Diese Handlungsfähigkeit zeigt sich demnach in dreifacher Hinsicht: im Sportunterricht, außerhalb der Schule und im späteren Leben der jungen Menschen. Basierend auf dem
SGK-Modell wurde ein Testinstrument für den Sportunterricht entwickelt und validiert (Töpfer, 2019). In jüngeren Veröffentlichungen wurde das SGK-Modell insbesondere mit dem Fokus des gesundheitsbezogenen Wissens und Verstehens in der Physical Literacy verortet (Strobl, Ptack, Töpfer, Sygusch, \& Tittlbach, 2020). Das Modell orientiert sich damit an der Denkweise, dass Wissen in der Physical Literacy sowohl im Bereich der kognitiven Komponenten als auch auf einer übergreifenden Ebene eine Schlüsselfunktion zukommt (Cale \& Harris, 2018; Ennis, 2015), indem es nicht nur als Basis für Performanzen gilt, sondern auch Ausgangspunkt für Transfer und Innovation darstellt.

Das BGK-Modell wiederum stammt ursprünglich aus dem Kontext der Rehabilitation (Pfeifer et al., 2013) und spiegelt die Notwendigkeit wider, neben klassisch orientierten physischen (z.B. motorischen Fähigkeiten und Fertigkeiten) und psychologischen Aspekten (z. B. Selbstwirksamkeit, Einstellungskomponenten) auch qualitative Komponenten des Bewegungsverhaltens (Steuerungskompetenz z.B. via Affektregulation oder Belastungssteuerung) in den Blick zu nehmen. Während Physical Literacy eine sehr inklusive Perspektive auf Bewegung und Sport einnimmt, lässt sich BGK eher als ein bewegungsbezogener Ansatz „im Dienste“ der Gesundheit charakterisieren (Carl, Sudeck, \& Pfeifer, 2020). Damit rückt dieser in die Nähe von Physical-Literacy-Studien, die die Wichtigkeit des Gesundheitsaspekts betonen (z. B. Cairney et al., 2019b; Cornish et al., 2020). Als ein weiterer wichtiger Unterschied auf der Ebene von Modellkomponenten ist hervorzuheben, dass körperliche Aktivität bei BGK konsequent als Ergebnisvariable geführt wird. Im Physical-Literacy-Ansatz hingegen fungiert körperliche Aktivität oft als separate Modellkomponente (Carl et al., 2020).

Insgesamt wird Physical Literacy im deutschsprachigen Raum vor allem im Rahmen von Interventionsstudien eingesetzt. Dabei wird Physical Literacy entweder explizit namentlich genannt (Demetriou, Bachner, Reimers, \& Göhner, 2018; Holler et al., 2019) oder 
zumindest in Bezug $\mathrm{zu}$ verwandten Konzepten (SGK und BGK) gesetzt (Bruland, Voß, Schulenkorf, \& Latteck, 2019; Strobl et al., 2020; Volk et al., 2021). In den zwei erstgenannten Studien dienen Indikatoren von Physical Literacy als Evaluierungskriterien. Zwar steht den Forscher ${ }^{\star}$ innen kein spezifisch validiertes Messinstrument zu Physical Literacy zur Verfügung, jedoch wird auf der Domänenebene auf etablierte Verfahren zurückgegriffen, wie beispielsweise auf Erhebungsinstrumente zur Motivation, zum Aktivitätsverhalten oder zum bewegungsbezogenen Wissen (Demetriou et al., 2018; Holler et al., 2019). Erste Forschungsprojekte mit einem Bezug zur Physical Literacy wurden in jüngster Zeit nicht nur in Deutschland (u.a. Demetriou et al., 2018; Wessely, Starke, Weyers, \& Joisten, 2021), sondern auch in Österreich (u.a. Holler et al., 2019) durchgeführt. So wurden beispielsweise im österreichischen Projekt „Primary Care \& Physical Literacy“ Trainer ${ }^{\star}$ innen in Gruppenkursen zu diesem Thema fortgebildet (Holler et al., 2021). Entsprechende Studien aus der deutschsprachigen Schweiz liegen uns nach aktuellen Recherchen nicht vor.

Resümierend lassen sich vier Beobachtungen herausarbeiten:

1. Es wird deutlich, dass Physical Literacy nur zögerlich in der Forschungslandschaft der deutschsprachigen Sportwissenschaft anzukommen scheint. Zum einen fließt Physical Literacy in konzeptionelle Arbeiten ein (u. a. Hummel \& Krüger, 2020), und zum anderen wird es als Grundlage für Forschungsprojekte herangezogen (u.a. Demetriou et al., 2018; Holler et al., 2019). Derzeit liegen im deutschsprachigen Raum jedoch noch keine weitreichenden Befunde zur Physical Literacy vor.

2. Auffällig ist, dass in den vorliegenden Publikationen meist versucht wird, die bereits vorhandenen deutschsprachigen Konzepte (SGK, BGK, körperliche Grundbildung) gewissermaßen nachträglich über Physical Literacy zu begründen, zu verorten oder teilweise zu erweitern (Carl et al., 2020; Hummel \& Krüger, 2020; Strobl et al., 2020).

Ger J Exerc Sport Res 2022 ·52:186-192 https://doi.org/10.1007/s12662-021-00754-2 (c) Der/die Autor(en) 2021

\section{Töpfer · J. Jaunig · J. Carl}

\section{Physical Literacy - to be discussed: eine Perspektive aus Sicht der deutschsprachigen Sportwissenschaft}

\section{Zusammenfassung}

Mit dem vorliegenden Beitrag möchten wir in der deutschsprachigen Sportwissenschaft zur Diskussion um das Konzept der Physical Literacy anregen. Im ersten Schritt soll dabei aufgezeigt werden, wie das Konzept der Physical Literacy aktuell sowohl international als auch im deutschsprachigen Raum rezipiert wird. Gerade mit Blick auf die deutschsprachige Sportwissenschaft möchten wir diesbezüglich einige Beobachtungen herausarbeiten. Es wird deutlich, dass Physical Literacy nur zögerlich in der Forschungslandschaft der deutschsprachigen Sportwissenschaft anzukommen scheint. Zudem ist erkennbar, dass gegenwärtige deutschsprachige Konzepte nachträglich über Physical Literacy begründet und erweitert werden. Die vorhandenen Entwürfe variieren dabei in ihrer inhaltlichen Schwerpunktsetzung. Darüber hinaus wird Physical Literacy in der deutschsprachigen Sportwissenschaft bislang kaum interdisziplinär diskutiert. Im zweiten Teil setzen wir an diesen Beobachtungen an und stellen Herausforderungen und Chancen zur Diskussion, warum sich die deutschsprachige Sportwissenschaft intensiver mit Physical Literacy beschäftigen sollte. In diesem Zusammenhang erkennen wir vier Herausforderungen. Dabei geht es darum, Physical Literacy begrifflich und konzeptionell zu deuten. Außerdem sollte die deutschsprachige Sportwissenschaft Physical Literacy interdisziplinär denken und entsprechend handeln. Die Auseinandersetzung mit Physical Literacy könnte zudem internationale Sichtbarkeit schaffen und bietet Impulse, Bewegungspraxis ganzheitlicher zu gestalten.

\section{Schlüsselwörter}

Bewegungskompetenz - Gesundheitskompetenz · Interdisziplinarität · Körperliche Aktivität $\cdot$ Kompetenz

\section{Physical literacy-to be discussed: a perspective from the point of view of German language sport science}

\section{Abstract}

This article is intended to encourage discussion about the concept of physical literacy in the German-speaking sport science community. The first step illustrates how the concept of physical literacy is currently embraced both internationally and in German-speaking countries. Particularly concerning German language sport science, we would like to elaborate on a few observations in this respect. It becomes clear that physical literacy only appears to hesitantly permeate the research landscape of German-speaking countries. In addition, it is discernible that current German language concepts are retrospectively substantiated and expanded through physical literacy. The existing drafts, however, vary in terms of their specific content focus. Furthermore, in German language sport science physical literacy has so far hardly been discussed in an interdisciplinary manner. In the second part, we take these observations as a starting point and discuss challenges and opportunities as to why German language sport science should deal more intensively with physical literacy. In this context, we identify four challenges. Physical literacy has to be discussed both conceptually and terminologically. In addition, German language sport science should pick up interdisciplinary ideas of physical literacy and react accordingly. The discussion of physical literacy could also promote international visibility and may inspire physically related practices in a more holistic manner.

\section{Keywords}

Health literacy · Physical competence . Interdisciplinarity - Physical activity . Competence
3. Im aktuell noch kleinen deutschsprachigen Diskurs zur Physical Literacy deutet sich bereits an, dass Physical Literacy durchaus verschieden ausgelegt und adaptiert wird. Dabei variieren die Überschneidungen zum ursprünglichen Ansatz von Whitehead je nach Entwurf. Vor dem Hintergrund der internationalen Diskussion verwundert dies nicht, da auch hier beispielsweise unterschiedliche Gewichtungen zugunsten von 
„physical competence“ (Hyndman \& Pill, 2018) oder „knowledge“ (Ennis, 2015) erkennbar sind.

4. Zudem wird deutlich, dass Physical Literacy bislang insbesondere sportpädagogisch (u.a. Strobl et al., 2020) und gesundheitswissenschaftlich (u. a. Carl et al., 2020) diskutiert wird. Eine breit gefächerte interdisziplinäre Auseinandersetzung liegt in der deutschsprachigen Sportwissenschaft aktuell nicht vor.

\section{Herausforderungen und Chancen: Warum sollte sich die deutschsprachige Sportwissenschaft intensiver mit Physical Literacy beschäftigen?}

$\mathrm{Zu}$ Beginn stellt sich die grundlegende Frage, wie man mit einem Konstrukt aus dem anglophonen Sprachraum umgeht, das gewissermaßen von außen in die deutschsprachige Sportwissenschaft hineingetragen wird. Ähnlich wie Lundvall (2015) für den internationalen Raum, sehen auch wir für die deutschsprachige Sportwissenschaft Herausforderungen und Chancen (• Abb. 1).

\section{Begrifflich und konzeptionell deuten}

Im ersten Schritt ist die Frage nach dem Umgang mit der Begrifflichkeit zu beleuchten. Zum einen ist es möglich, einen anglophonen Begriff schlichtweg in die deutsche Sprache zu übernehmen, wie es beispielsweise bei bestimmten Sportarten (etwa im Basketball) der Fall ist. In diesem Sinne wird in einem kürzlich veröffentlichten Praxishandbuch auch explizit von „Physical Literacy“ gesprochen (Holler et al., 2021). Zum anderen wird in der Regel versucht, ein deutschsprachiges Äquivalent für Physical Literacy zu finden. Mittlerweile befinden sich bereits verschiedene Wortschöpfungen in der deutschsprachigen Sportwissenschaft. So ist in den österreichischen Gesundheitszielen beispielsweise von „Bewegungskompetenz" die Rede (Delcour \& Griebler, 2016). Knisel und Bremer (2021) deuten zudem an, dass Physical Literacy auch gern als "physische Alphabetisierung“ verstanden wird. Schierz und Miethling (2017) erkennen in Physical Literacy eine sogenannte „Sportliteralität“. Im Gegensatz dazu deuten Hummel und Krüger (2020) sie als „neue körperliche Grundbildung“.

Dabei geht es nicht nur um die Frage der begrifflichen Übertragung, sondern auch um die inhaltliche Richtigkeit. Welche deutschsprachigen Konstrukte bilden ein passendes Pendant zur anglophonen Physical Literacy? Zumindest zeigen sich Schnittmengen zwischen Physical Literacy und dem deutschsprachigen Kompetenzdiskurs. Diesbezüglich schimmern auch Facetten der Handlungsfähigkeit (Gogoll \& Kurz, 2013) deutlich erkennbar im Konzept der Physical Literacy durch. Direkte Übersetzungen von Physical Literacy beispielsweise in „bewegungsbezogene Gesundheitskompetenz" (Joisten, 2020) erscheinen dabei jedoch ungenau, da sie einerseits selbst Physical Literacy nicht adäquat abbilden und anderseits zusätzlich Verwirrung zum (zugegebenermaßen) teils verwandten Konstrukt der Gesundheitskompetenz stiften, welches in der jüngeren Diskussion zunehmend ganzheitlicher auch im Kontext der zielgruppenorientierten Anwendung und sozialer Praktiken verstanden wird (Bröder, 2020). Insgesamt sind auch vor diesem Hintergrund weitere begriffliche Schärfungen nötig.

Neben den offenen Fragen zum begrifflichen Transfer liegen auf einer konzeptionellen Ebene auch mögliche Chancen in der intensiveren Rezeption der Physical Literacy im deutschsprachigen Raum. Insbesondere die Holistik des Physical-Literacy-Ansatzes findet international große Anerkennung (u.a. Pushkarenko et al., 2020) und bietet die Möglichkeit, tradierte Konzepte zu erweitern. Gerade auch der Kompetenzdiskurs im deutschsprachigen Raum könnte somit ausgeweitet und je nach Setting entsprechend ausgedeutet werden. Somit könnten erprobte Ansätze und Interventionen zur Bewegungsförderung verstärkt einfließen, die sich an der Lebenswelt und den Bedarfen der Menschen orientieren. In diesem Kontext sollte aber auch der übergreifenden Frage nachgegangen werden, welche Kompetenzfacetten für eine Teilhabe an Bewegung und Sport erforderlich sind. Welche Rolle spielen beispielsweise motorische Basiskompetenzen oder auch Wissen für die kulturelle Teilhabe (u.a. Herrmann, Gerlach, \& Seelig, 2016; Sygusch, Hapke, Liebl, \& Töpfer, im Druck)?

Der Diskurs um die begriffliche Deutung von Physical Literacy im deutschsprachigen Raum sollte dabei nicht nur einseitig von außen nach innen, sondern auch von von innen nach außen gerichtet sein. Demzufolge wäre zu prüfen, wie der deutschsprachige Diskurs beispielsweise um Bewegungsbildung, Kompetenzen und den Erziehenden Sportunterricht zunehmend in die internationale Physical-Literacy-Community hineingetragen werden kann.

\section{Interdisziplinär denken und handeln}

Die ersten Physical-Literacy-Publikationen, die der deutschsprachigen Sportwissenschaft zugeordnet werden können, lassen bereits erkennen, dass je nach sportwissenschaftlicher Disziplin eine andere Perspektive auf Physical Literacy eingenommen wird. Dies führt mitunter zu einer teils eindimensionalen Gewichtung ausgewählter Facetten.

Die Erforschung und Beschreibung von „physically literate individuals“ böte die Möglichkeit, unter der Annahme eines spezifischen Menschenbildes (Monismus, Existenzialismus, Phänomenologie; Pot, Whitehead, \& DurdenMyers, 2018) integrativ-interdisziplinäre Betrachtungsweisen voranzutreiben. Interdisziplinäre Perspektiven sind für die Sportwissenschaft geradezu typisch, laut Willimczik (2001) sogar vielmehr konstituierend für das Fach. Interdisziplinäre Diskussionen und Fragen nach der Vereinbarkeit sportwissenschaftlicher Teildisziplinen haben bereits in der Vergangenheit die deutsche Sportwissenschaft geprägt (u.a. Willimczik, 2001).

Mit Blick auf das Konzept der Physical Literacy bedeutet dies im Konkreten, dass es zur Belebung von "physical competence“, „knowledge and understanding“, „motivation and confidence“ sowie „dai- 


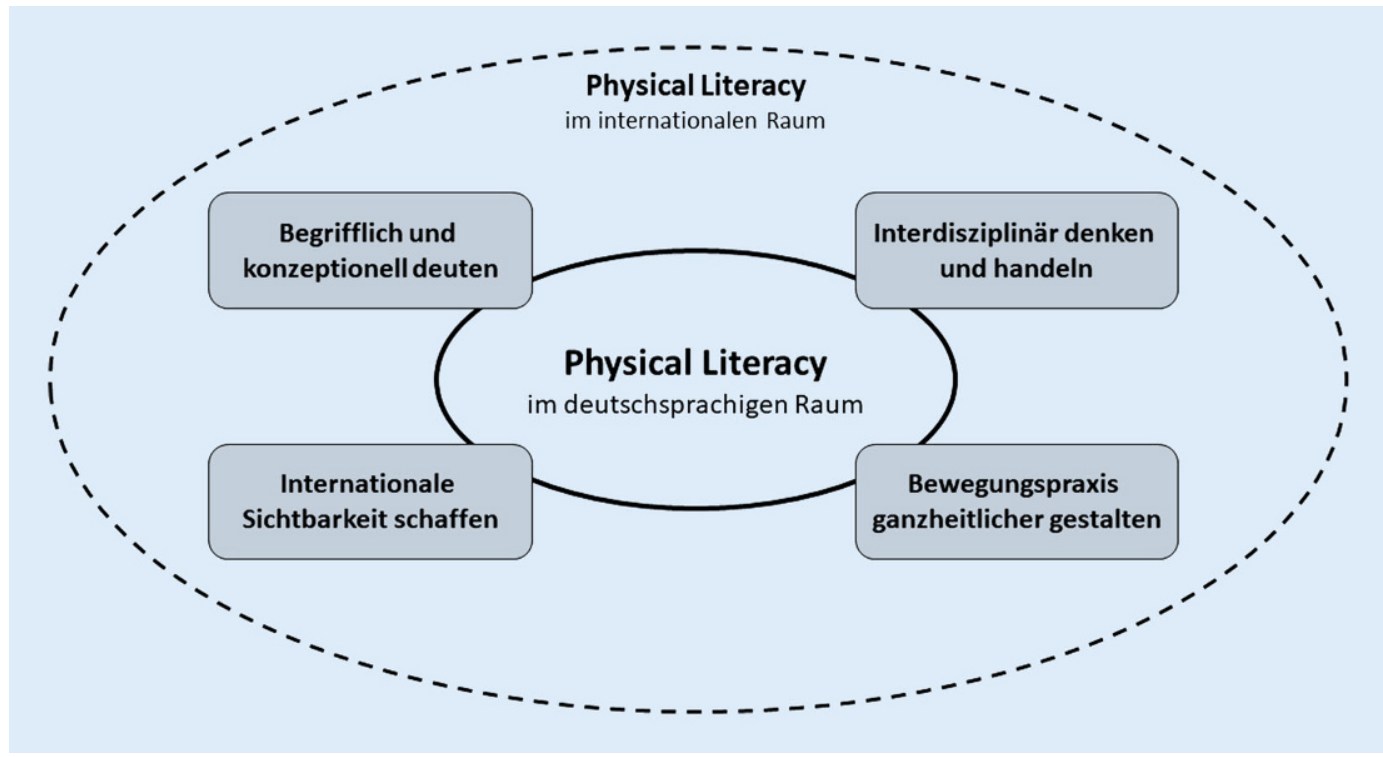

Abb. $1<$ Herausforderungen und Chancen von Physical Literacy im deutschsprachigen Raum ly behaviour" mindestens einer Konvergenz von Erkenntnissen aus den Feldern Sportmedizin, Trainingswissenschaft, Bewegungswissenschaft, Sportpsychologie und Sportpädagogik bedarf. Im Konzept selbst sind verschiedene Facetten enthalten, die sportwissenschaftlich aus Sicht verschiedener Disziplinen erforscht werden können. Physical Literacy kann dabei u. a. sportpsychologisch (z. B. Wissen, Motivation, Verhalten), sportpädagogisch (z.B. Bildung, Kompetenz, Verantwortungsübernahme), sportmotorisch (z.B. motorische Fähigkeiten und Fertigkeiten) und sportmedizinisch (z.B. Auswirkungen auf die physische Gesundheit) diskutiert werden.

\section{Internationale Sichtbarkeit schaffen}

Physical Literacy hat sich mittlerweile international etabliert. Vor diesem Hintergrund stellt sich die Frage, wie die deutschsprachige Sportwissenschaft von dem anglophonen Konzept der Physical Literacy profitieren kann. Für deutschsprachige Sportwissenschaftler ${ }^{*}$ innen bieten die vorhandenen internationalen (meist englischsprachigen) Publikationen zur Physical Literacy mittlerweile einen reichhaltigen (und sichtbaren) Wissensschatz. Gleichzeitig bleibt zu klären, wie die deutschsprachige Sportwissenschaft sich in der internationalen Physical-Literacy-Diskussion positio- nieren kann und will. Während beispielsweise aus anderen Ländern bereits übergreifende Berichte zur Physical Literacy dokumentiert sind (Whitehead, 2019), liegt aus dem deutschsprachigen Raum bislang kein landesspezifischer Bericht zum Status quo vor. Dementsprechend stellt sich die Frage, was die deutschsprachige Sportwissenschaft in die internationale Physical-LiteracyDiskussion einbringen kann: Welche Konzeptionen und Erkenntnisse aus dem deutschsprachigen Raum (z. B. zum Kompetenzdiskurs) könnten gegebenenfalls einen Mehrwert für die internationale Physical-Literacy-Diskussion bieten?

Zusammenfassend kann die Auseinandersetzung an Physical Literacy eine Sichtbarkeit der Forschung in beide Richtungen bieten. Zum einen wird die deutschsprachige Sportwissenschaft sichtbarer nach außen und beispielsweise stärker in internationalen Reviews berücksichtigt. Zum anderen bietet es ihr auch die Möglichkeit, sich international an vergleichbaren Forschungsarbeiten zu orientieren.

\section{Bewegungspraxis ganzheitlicher gestalten}

Das Physical-Literacy-Konzept ist nicht nur in der Forschung international weit verbreitet, sondern insbesondere im anglophonen Sprachraum auch in der
Praxis in Teilen fest verankert (u. a. Durden-Myers \& Whitehead, 2018). Die Grundidee fokussiert dabei die Förderung von integrativen Konzepten und Expert*innen für die Praxis. Vor diesem Hintergrund entstand auch das Praxishandbuch für Physical Literacy einer österreichischen Arbeitsgruppe (Holler et al., 2021). Darüber hinaus gilt es zu prüfen, wie überdies im Sinne einer ganzheitlichen Professionalisierung die Idee der Physical Literacy in die Praxis beispielsweise von Sporttherapeut*innen oder auch Sportlehrkräften getragen werden kann. Die deutschsprachige Sportwissenschaft könnte somit nicht nur aus Sicht der Forschung, sondern auch im Sinne der Praxis von dem Konzept profitieren.

\section{Fazit}

Wenn neue Konzepte in eine bestehende (hier deutschsprachige) Forschungslandschaft eingebracht werden, sind damit in der Regel erst einmal Irritationen und Skepsis verbunden. Begriffe müssen sortiert und adaptiert, konzeptionelle Unschärfen geklärt und empirische Grundlagen geschaffen werden. Ähnliche Herausforderungen und Chancen wurden in den vergangenen Jahren immer wieder auch in der internationalen Forschungslandschaft dokumentiert (Bailey, 2020; Tremblay et al., 2018). Dabei wird deutlich, dass (nur) über eine sukzessive Aus- 
einandersetzung mit dem Konzept der Physical Literacy auch dessen Potenziale und Grenzen erschlossen werden können. Genau diesen Weg sollte auch die deutschsprachige Sportwissenschaft gehen. Sie sollte sich offen und konstruktiv mit dem Konzept der Physical Literacy auseinandersetzen.

\section{Korrespondenzadresse}

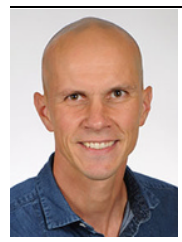

\section{Dr. Clemens Töpfer}

Institut für Sportwissenschaft, Friedrich-Schiller-Universität Jena

Seidelstraße 20, 07749 Jena,

Deutschland

clemens.toepfer@uni-jena.de

Funding. Open Access funding enabled and organized by Projekt DEAL.

Interessenkonflikt. C. Töpfer, J. Jaunig und J. Carl geben an, dass kein Interessenkonflikt besteht.

Open Access. Dieser Artikel wird unter der Creative Commons Namensnennung 4.0 International Lizenz veröffentlicht, welche die Nutzung, Vervielfältigung, Bearbeitung, Verbreitung und Wiedergabe in jeglichem Medium und Format erlaubt, sofern Sie den/die ursprünglichen Autor(en) und die Quelle ordnungsgemäß nennen, einen Link zur Creative Commons Lizenz beifügen und angeben, ob Änderungen vorgenommen wurden.

Die in diesem Artikel enthaltenen Bilder und sonstiges Drittmaterial unterliegen ebenfalls der genannten Creative Commons Lizenz, sofern sich aus der Abbildungslegende nichts anderes ergibt. Sofern das betreffende Material nicht unter der genannten Creative Commons Lizenz steht und die betreffende Handlung nicht nach gesetzlichen Vorschriften erlaubt ist, ist für die oben aufgeführten Weiterverwendungen des Materials die Einwilligung des jeweiligen Rechteinhabers einzuholen.

Weitere Details zur Lizenz entnehmen Sie bitte der Lizenzinformation auf http://creativecommons.org/ licenses/by/4.0/deed.de.

\section{Literatur}

Bailey, R. (2020). Defining physical literacy: making sense of a promiscuous concept. Sport in Society. https://doi.org/10.1080/17430437. 2020.1777104

Bröder, J. (2020). Health Literacy in der Kindheit und Jugend: Analyse des aktuellen Diskurses und damit verbundene Herausforderungen und konzeptionelle Überlegungen. In T. M. Bollweg, J. Bröder \& P. Pinheiro (Hrsg.), Gesundheit und Gesellschaft. Health Literacy im Kindes- und Jugendalter (S. 373-392). Wiesbaden: Springer. https://doi.org/10.1007/978-3-658-29816-6 22.

Bruland, D., Voß, M., Schulenkorf, T., \& Latteck, Ä.D. (2019). Mit Schwung und Energie durch den Tag. Partizipative Forschung zur Förderung der bewegungsbezogenen Gesundheitskompetenz bei Menschen mit Lernschwierigkeiten. Präven tion Und Gesundheitsförderung, 14(4), 368-374. https://doi.org/10.1007/s11553-019-00714-7.

Cairney, J. (Hrsg.). (2020). Special issue "Physical literacy in children and youth". International Journal of Environmental Research and Public Health, Bd. 17. https://www.mdpi.com/journal/ijerph/ special_issues/physical_literacy

Cairney, J., Dudley, D., Kwan, M., Bulten, R., \& Kriellaars, D. (2019b). Physical literacy, physical activity and health: toward an evidence-informed conceptual model. Sports Medicine, 49(3), 371-383. https://doi.org/10.1007/s40279-01901063-3.

Cairney, J., Kiez, T., Roetert, E. P., \& Kriellaars, D. (2019a). A 20th-century narrative on the origins of the physical literacy construct. Journal of Teaching in Physical Education, 38(2), 79-83. https://doi.org/ 10.1123/jtpe.2018-0072.

Cale, L., \& Harris, J. (2018). The role of knowledge and understanding in fostering physical literacy. Journal of Teaching in Physical Education, 37(3), 280-287. https://doi.org/10.1123/jtpe.20180134.

Carl, J., Sudeck, G., \&Pfeifer, K. (2020). Competencies for a healthy physically active lifestyle - reflections on the model of physical activity-related health competence. Journal of Physical Activity \& Health. https://doi.org/10.1123/jpah.20190442 .

Chen, A., \& Sun, H. (2015). A great leap of faith: editorial for JSHS special issue on physical literacy. Journal of Sport and Health Science, 4(2), 105-107. https://doi.org/10.1016/j.jshs.2015. 04.002 .

Cornish, K., Fox, G., Fyfe, T., Koopmans, E., Pousette, A. \& Pelletier, C. A. (2020). Understanding physical literacy in the context of health: a rapid scoping review. BMC Public Health, 20(1), 1569. https:// doi.org/10.1186/s12889-020-09583-8.

Delcour, J., \& Griebler, R. (2016). Gesundheitsziele Österreich. Machbarkeitsprüfung zu den Wirkungsziele-Indikatoren des GZ8. Wien: Gesundheit Österreich.

Demetriou, Y., Bachner, J., Reimers, A. K., \& Göhner, W. (2018). Effects of a sports-oriented primary school on students' physical literacy and cognitive performance. Journal of Functional Morphology and Kinesiology. https://doi.org/10. 3390/jfmk3030037.

Dudley, D., Cairney, J., \& Goodway, J. (2019). Special issue on physical literacy: evidence and intervention. Journal of Teaching in Physical Education, 38(2), 77-78. https://doi.org/10.1123/ jtpe.2019-0020.

Dudley, D., Cairney, J., Wainwright, N., Kriellaars, D., \& Mitchell, D. (2017). Critical considerations for physical literacy policy in public health, recreation, sport, and education agencies. Quest, 69(4), 436-452. https://doi.org/10.1080/ 00336297.2016.1268967.

Durden-Myers, E.J., \& Whitehead, M.E. (2018). Operationalizing physical literacy: special issue editorial. Journal of Teaching in Physical Education, 37(3), 234-236. https://doi.org/10. 1123/jtpe.2018-0130.

Edwards, L. C., Bryant, A. S., Keegan, R. J., Morgan, K., \& Jones, A.M. (2017). Definitions, foundations and association of physical literacy:a systematic review. Sports Medicine, 47(1), 113-126. https:// doi.org/10.1007/s40279-016-0560-7.

Edwards, L. C., Bryant, A. S., Keegan, R. J., Morgan, K., Cooper, S.-M., \& Jones, A. M. (2018). 'Measuring' physical literacy and related constructs: a systematic review of empirical findings. Sports Medicine, 48(3), 659-682. https://doi.org/10. 1007/s40279-017-0817-9.

Ennis, C. D. (2015). Knowledge, transfer, and innovation in physical literacy curricula. Journal of Sport and Health Science, 4(2), 119-124. https://doi.org/ 10.1016/j.jshs.2015.03.001.

Gogoll, A., \& Kurz, D. (2013). Kompetenzorientierter Sportunterricht - das Ende der Bildung? In H. Aschebrock \& G. Stibbe (Hrsg.), Didaktische Konzepte für den Schulsport (S. 79-97). Aachen: Meyer \& Meyer.

Gunnell, K. E., Longmuir, P.E., Barnes, J. D., Belanger, K. \& Tremblay, M.S. (2018). Refining the Canadian assessment of physical literacy based on theory and factor analyses. BMC Public Health, 18(Suppl 2), 1044. https://doi.org/10.1186/s12889-0185899-2.

Herrmann, C., Gerlach, E., \& Seelig, H. (2016). Motorische Basiskompetenzen in der Grundschule. Begründung, Erfassung und empirische Überprüfung eines Messinstruments. Sportwissenschaft,46(2), 60-73. https://doi.org/10.1007/ s12662-015-0378-8.

Holler, P., Jaunig, J., Amort, F.-M., Tuttner, S., HoferFischanger, K., Wallner, D., Simi, H., Müller, A., van Poppel, M. N. M., \& Moser, O. (2019). Holistic physical exercise training improves physical literacy among physically inactive adults: a pilot intervention study. BMC Public Health, 19(1), 393. https://doi.org/10.1186/s12889-019-6719-z.

Holler, P., Jaunig, J., Fink, S., Tuttner, S., Wallner, D., \& Amort, F. M. (2021). Physical Literacy. Holistik der Bewegungsförderung. Praxishandbuch. Wien: Österreichische Gesundheitskasse.

Hummel, A., \& Krüger, M. (2020). Physical Literacy und neue körperliche Grundbildung. Sportunterricht 69(7), 295-300. https://doi.org/10.30426/SU2020-07-2.

Hyndman, B., \& Pill, S. (2018). What's in a concept? A Leximancer text mining analysis of physical literacy across the international literature. European Physical Education Review, 24(3), 292-313. https://doi.org/10.1177/1356336X17690312.

International Physical Literacy Association (IPLA) (2017). Physical Literacy Definition. Zugriff unter https://www.physical-literacy.org.uk/

Joisten, C. (2020). Bewegung als Fundament einer gesunden Entwicklung von Kindern und Jugendlichen. In C. Breuer, C. Joisten \& W. Schmidt (Hrsg.), Vierter Deutscher Kinder- und Jugendsportbericht: Gesundheit, Leistung und Gesellschaft(S.78-98). Schorndorf: Hofmann.

Jurbala, P. (2015). What is physical literacy, really? Quest, 67(4), 367-383. https://doi.org/10.1080/ 00336297.2015.1084341.

Knisel, E., \& Bremer, M. (2021). Physical literacy in physical education. https://forschung-sachsenanhalt.de/project/physical-literacy-physicaleducation-24705.Zugegriffen: 2. Mai 2021.

Lundvall, S. (2015). Physical literacy in the field of physical education-A challenge and a possibility. Journal of Sport and Health Science, 4(2), 113-118. https://doi.org/10.1016/j.jshs.2015. 02.001.

Naylor, P.-J., \& Temple, V.A. (Hrsg.). (2018). Canadian assessment of physical literacy. BMC Public Health, Bd. 18 Supplement 2. https:// bmcpublichealth.biomedcentral.com/articles/ supplements/volume-18-supplement-2

Pfeifer, K., Sudeck, G., Geidl, W., \& Tallner, A. (2013). Bewegungsförderung und Sport in der Neurologie - Kompetenzorientierung und 
Nachhaltigkeit. Neurologie \& Rehabilitation, 19(1),7-19.

Pot, N., Whitehead, M. E., \& Durden-Myers, E. J. (2018). Physical literacy from philosophy to practice. Journal of Teaching in Physical Education, 37(3), 246-251. https://doi.org/10.1123/jtpe.20180133.

Pushkarenko, K., Causgrove Dunn, J., \& Wohlers, B. (2020). Physical literacy and inclusion: a scoping review of the physical literacy literature inclusive of individuals experiencing disability. PROSPECTS, 50(1/2), 107-126. https://doi.org/ 10.1007/s11125-020-09497-8.

Roetert, E. P., \& MacDonald, L.C. (2015). Unpacking the physical literacy concept for K-12 physical education: What should we expect the learner to master? Journal of Sport and Health Science, 4(2), 108-112. https://doi.org/10.1016/j.jshs.2015. 03.002 .

Schierz, M., \& Miethling, W.-D. (2017). Sportlehrerprofessionalität: Ende einer Misere oder Misere ohne Ende? German Journal of Exercise and Sport Research,47(1), 51-61. https://doi.org/10.1007/ s12662-017-0440-9.

Scott, J. J., Hill, S., Barwood, D., \& Penney, D. (2020). Physical literacy and policy alignment in sport and education in Australia. European Physical Education Review. https://doi.org/10. 1177/1356336X20947434.

Strobl, H., Ptack, K., Töpfer, C., Sygusch, R., \& Tittlbach, S. (2020). Effects of a participatory schoolbased intervention on students' health-related knowledge and understanding. Frontiers in Public Health, 8, 122. https://doi.org/10.3389/ fpubh.2020.00122.

Sudeck, G., \& Pfeifer, K. (2016). Physical activityrelated health competence as an integrative objective in exercise therapy and health sports - conception and validation of a short questionnaire. Sportwissenschaft, 46(2), 74-87. https://doi.org/10.1007/s12662-016-0405-4.

Sygusch, R., Hapke, J., Liebl, S., \& Töpfer, C. (Hrsg.). (in Druck). Kompetenzorientierung im Sport. Grundlagen, Modellentwurf und Anwendungsbeispiele. Schorndorf:Hofmann.

Töpfer, C. (2019). Sportbezogene Gesundheitskompetenz: Kompetenzmodellierung und Testentwicklung für den Sportunterricht. Schriften der Deutschen Vereinigung für Sportwissenschaft, Bd. 286. Hamburg: Feldhaus.

Töpfer, C., \&Sygusch, R. (2014).Gesundheitskompetenz im Sportunterricht. In S. Becker (Hrsg.), Research. Aktiv und gesund? Interdisziplinäre Perspektiven auf den Zusammenhang zwischen Sport und Gesundheit (S. 153-179). Wiesbaden: Springer VS.

Tremblay, M.S., Costas-Bradstreet, C., Barnes, J.D., Bartlett, B., Dampier, D., Lalonde, C., Leidl, R. Longmuir, P., McKee, M., Patton, R., Way, R., \& Yessis, J. (2018). Canada's physical literacy consensus statement: process and outcome. BMC Public Health, 18(Suppl 2), 1034. https://doi. org/10.1186/s12889-018-5903-x.

UNESCO (2015). International charter of physical education, physical and sport.Zugriffam 9.9.2021 unter https://unesdoc.unesco.org/ark:/48223/ pf0000235409

Volk, C., Rosenstiel, S., Demetriou, Y., Krustrup, P., Thiel, A., Trautwein, U., Wagner, W., Höner, O., \& Sudeck, G. (2021). Effects of a physical education intervention programme for ninthgraders on physical activity-related health competence: findings from the GEKOS cluster randomised controlled trial. Psychology of Sport
\& Exercise.https://doi.org/10.1016/j.psychsport. 2021.101923.

Wessely, S., Starke, D., Weyers, S., \& Joisten, C. (2021). Closing the gap between practice and science in school- and community-based participatory physical literacy promotion: study protocol of the StuPs project. BMC Public Health, 21(1), 642. https://doi.org/10.1186/s12889-021-10666-3.

Whitehead, M. (2001). The concept of physical literacy. European Journal of Physical Education, 6(2), 127-138. https://doi.org/10.1080/ 1740898010060205.

Whitehead, M. (Hrsg.). (2019). Physical literacy across the world. Abingdon: Routledge.

Willimczik, K. (2001). Geschichte, Struktur und Gegenstand der Sportwissenschaft (1. Aufl.). Sportwissenschaft interdisziplinär: Ein wissenschaftstheoretischer Dialog, Bd. 1. Hamburg: Czwalina. 